\title{
Passive Source Localization in a Randomly Distributed Wireless Sensor Networks
}

\author{
Muhammad Tabish Niaz \\ NUST (MCS) \\ Pakistan
}

\author{
Dr. Adnan Ahmad Khan \\ CASE \\ Pakistan
}

\author{
Dr. Imran Shafi \\ CASE \\ Pakistan
}

\begin{abstract}
This paper proposes a source localization scheme using random arrays of Wireless Sensor Networks (WSN). A Total Least Square (TLS) estimator is proposed which improves the result of the location of source node. Using a relatively new Direction of Arrival (DOA) estimation technique Space Division Multiple Access (SDMA) receiver the proposed solution is able to perform localization in a multipath environment. The propose scheme considers both Line of Sight (LOS) and Non Line of Sight (NLOS) signals to perform the localization with the TLS estimator which is efficient than a simple Least Square (LS) estimator. Simulation results are included to demonstrate that the proposed solution provides an improved estimate by exploiting the NLOS information, SDMA receiver and using TLS estimator.
\end{abstract}

\section{Keywords}

Localization, wireless sensor networks, DOA, TDOA, SDMA receiver, Least square, Total Least Square, Singular Value Decomposition, Estimator, MUSIC algorithm.

\section{INTRODUCTION}

WSN has gained a lot of popularity in recent years that much advancement is being made in this field. One of the advancement that has caught attention is the localization problem in WSN. Localization is the process of finding the location of a source. There are number of wireless location estimation schemes, and they are broadly classified according to whether they take a geometrical or statistical approach. Under the geometrical approach, the geometric relationship between the source node and the reference is exploited to establish the Euclidean distance between them and that is used to find the physical location of the source node. The information that is used to measure the location can be the DOA, Time of Arrival (TOD) [1] and Time Difference of Arrival (TDOA) [2]. In the statistical approach, the measured data is usually the Received Signal Strength (RSS) [3] [4] [5]. In this paper the geometrical approach is used to find the location of a source node.

Two primary approaches in geometrical localization that have evolved are DOA and TDOA. In DOA localization the antenna elements examine the incident signals to obtain the bearing estimates. The bearing estimates are used for source node location determination using triangulation [6]. TDOA based localization system estimate the source location using the intersection of the hyperboloids, which are the set of range difference measurements between three or more receiving sensor [7].

Accurate localization requires the knowledge of the spatial characteristic of the wireless channel since these characteristic can significantly affect the performance of the localization. Thus a system is required which can efficiently model the wireless channel and accurately predict the channel response [8]. This complex propagation phenomena lead to uncertainty in deciding whether the received signal is a LOS or it is the reflection [9]. This problem can lead to significant errors when locating a source node. This problem gets more severe when there is no LOS signal present.

In the literature there are several proposals that consider the presence of NLOS signal. The first category is comprised of schemes which attempt to mitigate the effect of NLOS signal. Only the LOS signal was considered in the measurements ignoring the NLOS. The second category identifies the arrays receiving the NLOS signal and ignoring them from the calculation. In both of the approaches the authors try to minimize the impact of the NLOS signals instead of using them [10] [11] [12] [13] [14] [15] [16]. Recently, a third category of solution has emerged which attempts to exploit the use of NLOS signals [17] [18].

This paper proposes a source localization scheme which considers both LOS and NLOS signals. The DOA are collected using a recently new SDMA receiver [19] and then using TLS [20] an estimation of the location is determined. In this paper both DOA and TDOA are used to find the location of the source node. The DOA gives the bearings and using TDOA we can make a source and reflector pair which further helps in determining the location of the source node.

The organization of the paper is as follows. In Section 2, a LS estimator is compared with the TLS. In section 3, the use of SDMA receiver for obtaining bearing is explained. In section 4, the proposed localization scheme is discussed. In section 5, simulation results are provided to analyze and compare with the existing solution. Section 6, concludes the paper.

\section{TOTAL LEAST SQUARE ESTIMATOR}

The least square estimation scheme is used to be the easiest and the cheapest solution to perform the localization from DOA and TDOA measurements. Despite this fact, there are many errors present in the measurement of the DOA and TDOA which may significantly introduce errors in the estimation of the source location. The LS estimator performs the same as Maximum likelihood estimator if the noise present is Gaussian distributed.

The estimators proposed are based on the geometric configuration of the Fig.1. There are $\mathrm{K}$ arrays and each array obtains the DOA estimates by processing the incoming signal from the source. $\Theta_{\mathrm{k}}$ is the bearing obtained by $\mathrm{k}^{\text {th }}$ array. 


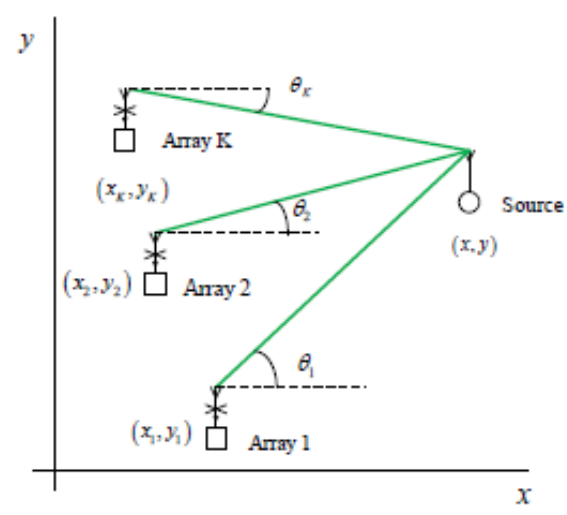

Figure 1 System Configuration

\subsection{Least Square Estimator}

The formulation of the LS problem based on the configuration of Figure. 1 can be shown as

$$
\left[\begin{array}{cc}
1 & \tan \left(\theta_{1}\right) \\
1 & \tan \left(\theta_{2}\right) \\
\vdots & \vdots \\
1 & \tan \left(\theta_{k}\right)
\end{array}\right]\left[\begin{array}{l}
y \\
x
\end{array}\right]=\left[\begin{array}{c}
y_{1}-\tan \left(\theta_{1}\right) \\
y_{2}-\tan \left(\theta_{2}\right) \\
\vdots \\
y_{k}-\tan \left(\theta_{k}\right)
\end{array}\right]
$$

or

$$
A(\theta) \bar{w}=\bar{b}(\theta)
$$

The matrix $A(\theta)$ has a specific structure and is referred as a Vandermonde matrix. The solution of the LS problem involving such a matrix is known to be computationally efficient and accurate [21]. Solving (2), we arrive at [22].

$$
\bar{w}=A^{+}(\theta) \bar{b}(\theta)
$$

where

$$
A^{+}(\theta)=\left[A^{T}(\theta) A(\theta)\right]^{-1} A^{T}(\theta)
$$

is the pseudo inverse of $A(\theta)$.

\subsection{Total Least Square Estimator}

The TLS solution performs better because it takes into the factors that are ignored by the LS. The matrix $A$ and $b$ are affected by errors [20]. These errors that affect the estimates can be modeling errors, system errors, channel errors, errors due to noise, and etc. The TLS takes into consideration these errors and provides a solution that is more accurate than the LS.

Let

$$
\begin{aligned}
& C=\left[\begin{array}{ll}
A & \bar{b}
\end{array}\right] \\
& \text { where } C \in C^{k \times 3}
\end{aligned}
$$

Using singular value decomposition (SVD) on the matric $\mathrm{C}$ we get

$$
C=U \Sigma V^{H}
$$

where $U \in C^{k \times 3}, \Sigma \in R^{3 \times 3}$ and $V \in C^{3 \times 3}$. Matrices $\mathrm{U}$ and $\mathrm{V}$ are unitary and $\Sigma$ is a diagonal matrix of the form

$$
\Sigma=\left[\begin{array}{ccc}
\sigma_{1 C} & 0 & 0 \\
0 & \sigma_{2 C} & 0 \\
0 & 0 & \sigma_{3 C}
\end{array}\right]
$$

where the diagonal elements are the singular values of $\mathrm{C}$, satisfying

$$
\sigma_{1 C} \geq \sigma_{2 C} \geq \sigma_{3 C}
$$

The TLS solution of the least square problem using TLS estimator is given by [20]

$$
\bar{x}_{T L S}=\left(A^{H} A-\sigma_{3 c} I\right)^{-1} A^{H} \bar{b}
$$

\section{SDMA RECEIVER}

The first step in localization is to find the bearing of the source node. There are many algorithm currently proposed in literature. Maximum-likelihood (ML) is always the optimum solution but using that in wireless network especially in WSN is a waste of energy. The ML is not accepted because of its high computational complexity. The Multiple Signal Classification (MUSIC) algorithm is perhaps the one of the most popular suboptimal techniques present out in the literature. It provides super resolution DOA estimation in a spatial pseudo spectral plot by utilizing the orthoganality between the signal and the noise subspaces.

A relatively new method of determining the DOA's is introduced in [19]. The use of SDMA receiver can be used in measuring the bearings of a source node. The SDMA receiver does not rely on the subspace decomposition of the correlation matrix as in the case of MUSIC algorithm, rather it crosscorrelates the received signal with a pre-computed set of array responses for every direction of interest. Due to this the computation cost is far less than MUSIC and the accuracy is more.

\subsection{SDMA Algorithm}

From Figure 2, consider a sensor array of size K. The output of each array element $\overline{x_{n}}(t)$ is phase-modulated by a set of uncorrelated spreading sequences $\overline{w_{n}}(t)$.. These sequences can be any type of pseudo random or orthogonal sequence. The produced array outputs are then orthogonal or nearly orthogonal. In matrix notation, the output of the array is

$$
Y=W^{H} X
$$

where $X$ is the incident signal (11) and $W \in C^{N \times L}$ is the matrix of the spreading sequences (12).

$$
\begin{gathered}
X=A(\theta) S+N \\
W=\left[\begin{array}{llll}
\overline{w_{1}}(t) & \overline{w_{2}}(t) & \ldots & \overline{w_{N}}(t)
\end{array}\right]^{H}
\end{gathered}
$$


The signals stored in the virtual array are also modulated by the same set of spreading sequences. In matrix notation, the output of the virtual array is given as

$$
V=W^{H} A_{M}(\theta)
$$

where $A_{M}(\theta) \in C^{N \times L}$ is the matrix of the array responses of all sensors for all DOA's. The correlator cross-correlates the array signal with that of the output of the virtual array as

$$
R=V^{H} Y
$$

where $\mathrm{R}$ is $\mathrm{K} \times \mathrm{L}$ matrix. The spatial spectrum of the SDMA receiver is then

$$
P_{S D M A}=\left|R_{k}\right|
$$

where

$$
R_{S}=\left[\sum_{I=1}^{L} R(1, l) \quad \sum_{l=1}^{L} R(2, l) \quad \cdots \quad \sum_{l=1}^{L} R(K, l)\right]
$$

The peaks of $\mathrm{P}_{\mathrm{SDMA}}$ correspond to the DOAs of the incident signals.

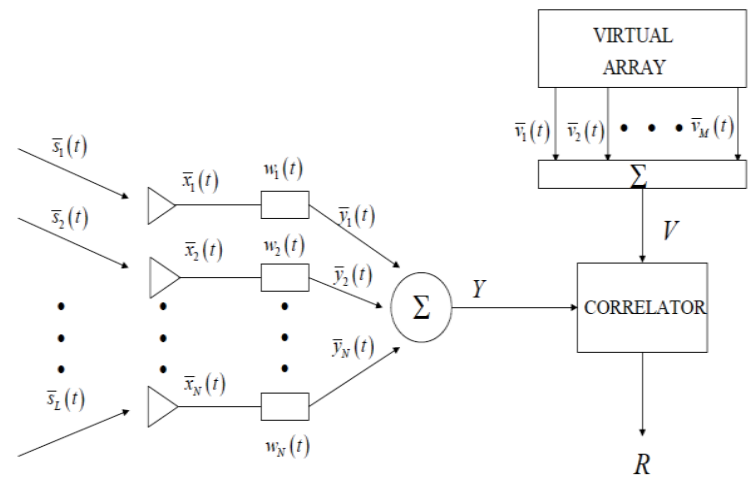

Figure 2 SDMA Receiver [From [23]]

\subsection{SDMA vs. MUSIC}

The SDMA receiver does not compute the correlation matrix, but just correlates the received signal with a pre computed one. This in contrast with the MUSIC algorithm does not rely on the correlation matrix. The SDMA receiver does not require knowledge of the number of incident signals compared with MUSIC algorithm, where number of incident signal are required before the computation is carried out. Finally, the SDMA receiver does not rely on any complex adaptive or slow iterative methods. It only compares the received value with the precomputed value. The results are given in section 5

\section{PROPOSED SCHEME}

The proposed localization scheme is implemented in three steps. The following Figure 3 is the block diagram of the proposed localization scheme.

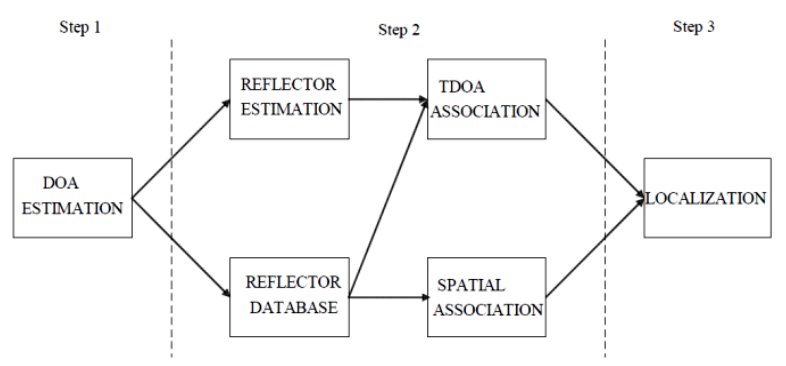

Figure 3 Proposed Localization Scheme

\subsection{DOA estimation}

The DOA estimation is done is step1 and this is performed by the SDMA receiver that is discussed in Section 3. The random array is used to implement the SDMA receiver because random arrays do not suffer from grating lobes. They are also not limited to a maximum sensor spacing of 0.5 wavelengths. This is an advantage because with smaller number of sensor nodes we can cover large area. Other than this random array are more flexible in a mobile environment. The bearings are obtained by the SDMA receiver and they are compared with the pre obtained bearing stored in the virtual array.

\subsection{Bearing Association}

Once we have the bearing the next step is to associate them to a source reflector pair. For each source reflector pair, a LOS and a NLOS is identified based on the knowledge of the reflector position and geometry. This reflector knowledge can be obtained by static databases which have the footprint of the large objects present in the environment or they can be dynamically be created by sending a beacon from a known location. Using this database we can associate the source with a reflector by measuring the bearing and the time stamp given by the TDOA calculation.

\subsection{Localization}

Once the source reflector database is made step 3 is used to run the localization scheme. From the second step we get the position of the reflector and its orientation we can find the location of a source. The localization is done by the TLS estimator proposed in Section 2. The matrix $A$ and $b$ mentioned before in section 2 will be as follows

$$
\begin{gathered}
A=\left[\begin{array}{cc}
1 & -\tan \theta_{L} \\
1 & -\tan \left(2 \theta_{o}-\theta_{R}\right.
\end{array}\right], \\
\bar{b}=\left[\begin{array}{c}
y_{A}-\tan \theta_{L} x_{A} \\
-\tan \left(2 \theta_{o}-\theta_{R}\right) x_{A}
\end{array}\right]
\end{gathered}
$$

The formulation of (17) can readily be expanded for multiple reflectors. We consider two approaches when the bearings from multiple arrays are available. The first is a centralized solution in which we formulate the TLS problem by considering the bearings of all arrays together. The second approach is distributed which solves the localization problem for each array estimates. The latter approach is of particular interest in wireless sensor network deployment since it distributes the processing load among multiple sensor nodes and reduces the communication burden which is the primary energy consumer. 


\section{RESULTS}

All the simulations were performed in Matlab. The system configuration is that, 3 to 7 randomly distributed arrays were used which cover an area of $2025 \mathrm{~m}^{2}$, centered at the origin. A single source is considered which is transmitting a narrow band signal with $30 \mathrm{kHz}$ bandwidth at a carrier frequency of $300 \mathrm{MHz}$. the signal is received via two multipath components. The first path is the LOS and the second path is a NLOS 'single bounce' signal. The power of the transmitted signal is $0.1 \mathrm{~mW}$. The metric used is the root mean square error (RMS), defined as $\gamma=\sqrt{\sigma^{2}+\mu^{2}}$ where $\sigma^{2}$ is the error variance and $\mu$ is the error mean. 75 Monte Carlo simulations were performed and the results are summarized as follows.

The Figures 4 and 5 are the comparison of the two algorithms MUSIC and SDMA to find the DOA of the incident signal. From the two figures we can clearly state that the SDMA algorithm performs better than MUSIC. The SDMA provided steep lobes while the MUSIC lobes are not steep and they are difficult to identify. The accuracy of the SDMA hence is better than that of the MUSIC. Hence it is clearly evident that SDMA should be used for the localization process. The cross correlation magnitudes of both algorithms are shown in the Figure 4 and 5 . The cross correlation magnitude of SDMA reliever is less than the magnitude of the MUSIC algorithm which is a positive sign as we can identify the DOA easily. Secondly by increasing the number of sensor in an array further improves the result when we compare Figure 4 and 5. From the Figure 4 we can calculate that the mean correlation magnitude of MUSIC is 0.7 and the SDMA has a magnitude of 0.45 . If we increase the number of sensors we can further improve the average correlation magnitude of MUSIC from 0.7 to 0.62 and of SDMA from 0.45 to 0.31 . this can be seen from Figure 5 .

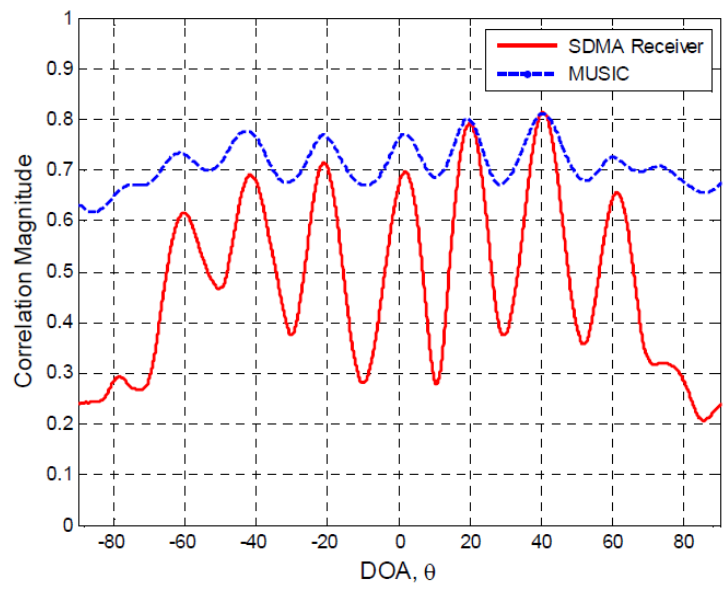

Figure 4 Comparison of MUSIC and SDMA for random arrays of 50 sensors with an area of $25 \mathbf{~ m}^{2}$

The proposed localization scheme outperforms the LOS only based localization scheme shown in figure 6 . This is because more bearing are available to perform the localization better. With the proper conditioning the LS performed better then the only LOS localization but TLS comes out to be the best solution. It has managed to eliminate the errors present in the matrix $A$ and $b$.
The figure 7 is comparison of the two estimators when they are applied to a scenario in which the reflector is unknown. Here it is evident that still TLS performs better than the LS algorithm.

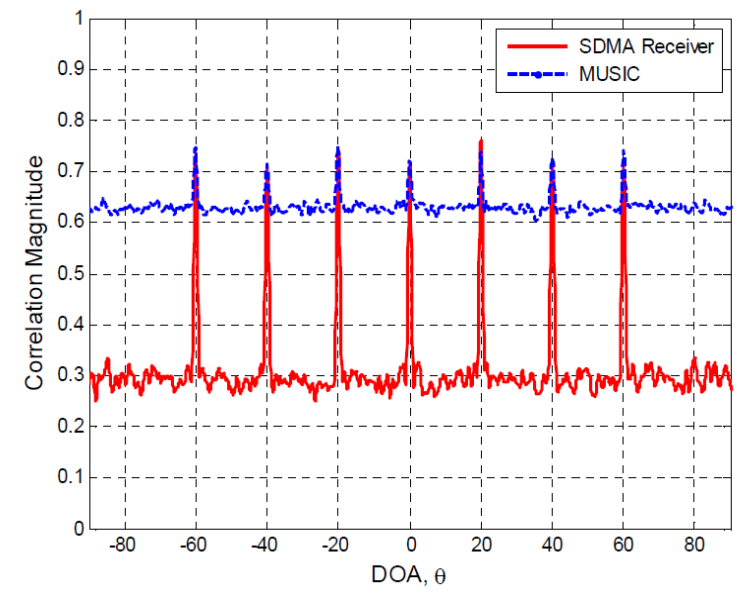

Figure 5 Comparison of MUSIC and SDMA for random arrays of 50 sensors with an area of $2500 \mathrm{~m}^{2}$

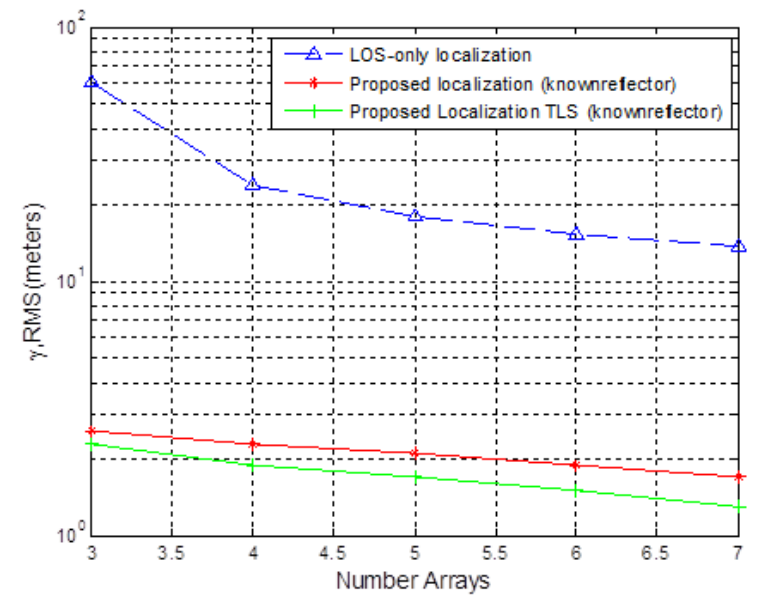

Figure 6 Comparison of LOS and NLOS using both LS and TLS estimator on a known reflector

\section{CONCLUSION}

This paper proposes a passive source localization scheme which exploits the NLOS signals from a non- cooperative source. The proposed solution is comprised of three parts: DOA estimator, bearing association, and the source localization scheme. The use of SDMA was shown to provide better results than using MUSIC algorithm. And applying TLS provide far better result than using a LS estimator. Simulation results were provided to demonstrate the accuracy of the proposed scheme and the use of SDMA receiver and TLS estimator further improve the current result of the existing literature. 


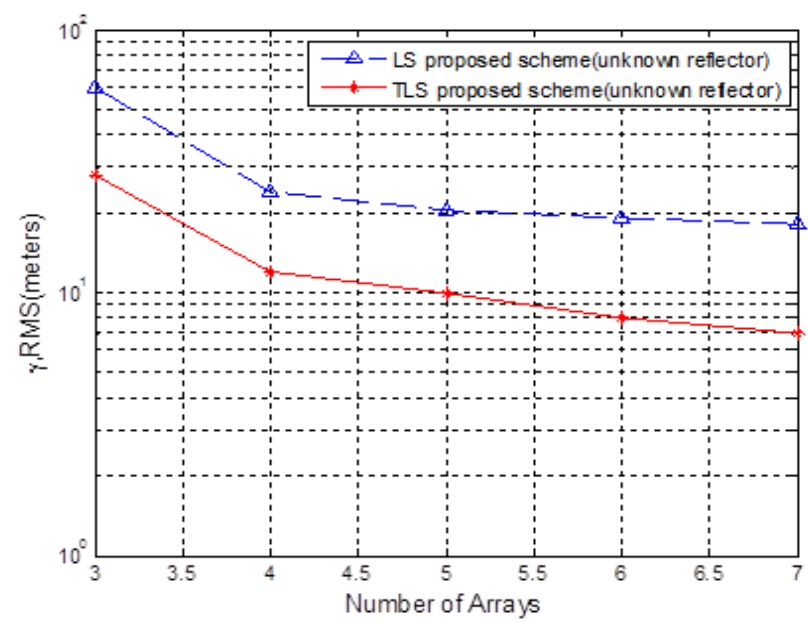

Figure 7 Comparison of LS and TLS estimator on an unknown reflector

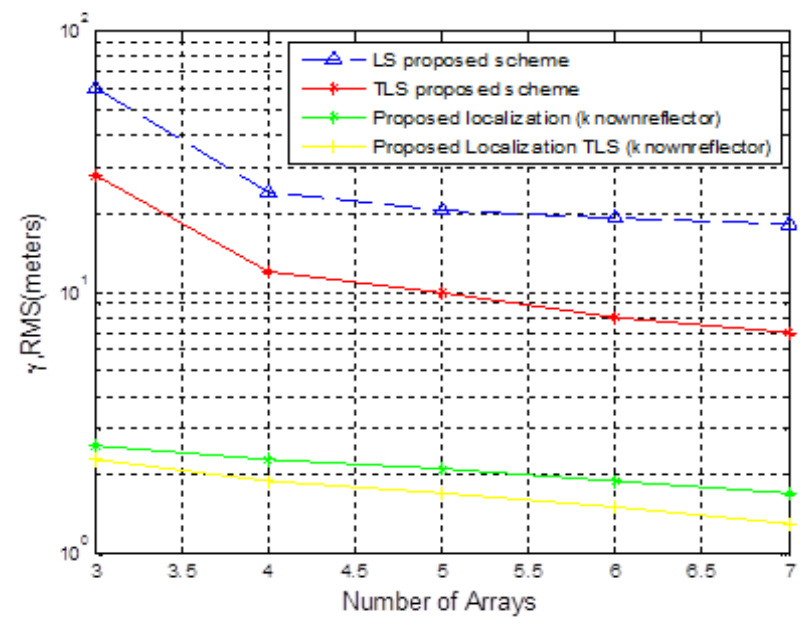

Figure 8 Comparison of LS and TLS on both known and unknown reflectors

\section{REFERENCES}

[1] X. Wang, Z.X. Wang, and B.O'. Dea, "A TOA-Based Location Algorithm Reducing the Errors Due to Non-Lineof-Sight (NLOS) Propagation," IEEE Trans. Vehicular Technology, vol. 52, pp. 112-116, Jan. 2003.

[2] Y.T. Chan and K.C. Ho, "A Simple and Efficient Estimator for Hyperbolic Location," IEEE Trans. Signal Processing, vol. 42, pp. 1905-1915, Aug. 1994.

[3] T. Roos, P. Myllymaki, and H. Tirri, "A Statistical Modeling Approach to Location Estimation," IEEE Trans. Mobile Computing, vol. 1, no. 1, pp. 59-69, Jan.-Mar. 2002.

[4] Z.L. Wu, C.H. Li, J.K.Y. Ng, and K.R.P.H. Leung, "Location Estimation via Support Vector Regression," IEEE Trans. Mobile Computing, vol. 6, no. 3, pp. 311-321, Mar. 2007.

[5] T. Ross, P. Myllymaki, H. Tirri, P. Misikangas, and J. Sievanen, "A Probabilistic Approach to WLAN User Location Estimation," Int'l J. Wireless Information Networks, vol. 9, no. 3, July 2002.
[6] R. A. Poisel, Electronic Warfare Target Location Methods. Norwood, MA: Artech House, 2005.

[7] J. C. Liberti, T. S. Rappaport, Smart Antennas for Wireless Communications: IS-95 and Third Generation CDMA Applications. Upper Saddle River, NJ: Prentice Hall, 1999

[8] R. Ertel et al., "Overview of Spatial Channel Models for Antenna Array Communications Systems." IEEE Trans. on Personal Communications, Feb 1998, vol. 5, pp 10-22.

[9] J. C. Chen, K. Yao, R. E. Hudson, "Source Localization and Beamforming." IEEE Signal Processing Magazine, Mar 2002, vol. 19, pp 30-39.

[10] P.C. Chen, "A Non-Line-of-Sight Error Mitigation Algorithm in Location Estimation," Proc. IEEE Wireless Comm. Networking Conf. (WCNC '99), vol. 1, pp. 316320, 1999.

[11] S. Venkatraman, J. Caffery, and H.R. You, "Location Using LOS Range Estimation in NLOS Environments," Proc. 55th IEEE Vehicular Technology Conf. (VTC Spring '02), vol. 2, pp. 856-860, May 2002.

[12] N. Khajehnouri and A.H. Sayed, "A Non-Line-of-Sight Equalization Scheme for Wireless Cellular Location," Proc. IEEE Int'l Conf. Acoustics, Speech, and Signal Processing (ICASSP '03), vol. 6, pp. 549-552, Apr. 2003.

[13] J. Borras, P. Hatrack, and N.B. Mandayam, "Decision Theoretic Framework for NLOS Identification," Proc. 48th IEEE Vehicular Technology Conf. (VTC '98), vol. 2, pp. 1583-1587, 1998

[14] L. Xiong, "A Selective Model to Suppress NLOS Signals in Angle of Arrival (AOA) Location Estimation," Proc. Ninth IEEE Int'l Symp. Personal, Indoor, and Mobile Radio Comm. (PIMRC '98), vol. 1, pp. 461-465, 1998.

[15] L. Cong and W.H. Zhuang, "Nonline-of-Sight Error Mitigation in Mobile Location," IEEE Trans. Wireless Comm., vol. 4, pp. 560-572, Mar. 2005.

[16] Y.T. Chan, W.Y. Tsui, H.C. So, and P.C. Ching, "Time-ofArrival Based Localization under NLOS Conditions," IEEE Trans. Vehicular Technology, vol. 55, pp. 17-24, Jan. 2006.

[17] J. Li, J. Conan, and S. Pierre, "Mobile Station Location Estimation for MIMO Communication Systems," Proc. Third Int'l Symp. Wireless Comm. Systems (ISWCS '06), Sept. 2006.

[18] H.L. Miao, K. Yu, and M.J. Juntti, "Positioning for NLOS Propagation: Algorithm Derivations and Cramer-Rao Bounds," Proc. IEEE Int'l Conf. Acoustics, Speech, and Signal Processing (ICASSP '06), vol. 4, pp. 1045-1048, June 2006

[19] C. Elam, " Method and Apparatus for Space Division Multiple Access Receiver," Patent No.6,823,021, Rights assigned to in Greenwich Technology Associates, One Soundview Way, Danen, CT. Nov 23, 2004.

[20] G. H. Golumb, C. F. Van Loan, An Analysis of the Total Least Square problem, SIAM, 1980

[21] G. H. Golumb, C. F. Van Loan, Matrix Computations, The Johns Hopkins University Press, Baltimore, 1996.

[22] L. N. Trefethen, D. Bau III, Numerical Linear Algebra, SIAM, Philadelphia, 1997

[23] F. Gross, Smart Antennas for wireless communication, New York, NY: Mc Graw Hill, 2005 